\title{
AN INTERDIGITATED GATE ELECTRODE FIELD EFFECT TRANSISTOR (IGEFET) FOR EPOXY CURE MONITORING
}

\author{
John M. Wiseman, Captain, USAF \\ Air Force Institute of Technology (AFIT/ENG) \\ Wright-Patterson AFB, OH 45433-6583 \\ Edward S. Kolesar, PhD, Lt Col, USAF \\ Air Force Institute of Technology (AFIT/ENG) \\ Wright-Patterson AFB, OH $45433-6583$
}

\section{ABSTRACT}

An interdigitated gate electrode field effect transistor (IGEFET) has been fabricated and utilized to monitor the cure of a common epoxy. The IGEFET sensor consists of an interdigitated gate electrode structure which is coupled to the gate of a conventional metal-oxidesemiconductor field-effect transistor (MOSFET). The epoxy was deposited on the interdigitated gate electrode and the IGEFET's electrical performance was observed as the epoxy cured. The crosslinking chemical reaction during epoxy cure caused impedance perturbations that were quantified when the IGEFET was excited with a voltage pulse. Charge transferred through the chemically-active epoxy is manifested as a temporally-dependent potential applied to the MOSFET gate contact. By operating the MOSFET as a linear amplifier, a potential corresponding to the temporally-dependent gate voltage was directly measured at the amplifier output. The Fourier transform of the IGEFET's time-domain response at specific time increments was computed. The resulting epoxy cure spectra were compared to a common baseline formed by computing the difference spectra involving the IGEFET's pulse excitation signal and the signal corresponding to the epoxy's chemical state at a specific instant of time. The abundance of difference spectral components yields valuable information for determining the chemical state of the epoxy's cure.

\section{INTRODUCTION}

Composite materials have recently become a critical engineering staple which are increasingly replacing equivalent strength metals while yielding significant weight reductions [1]. Although research concerning these materials is approximately 25 years old, their recent evolutionary usage and product experience has motivated a comprehensive scientific investigation of their processing characteristics. Composite materials are inherently homogeneous, and they are comprised of a matrix material (polymeric or metallic), a reinforcing fiber (polymeric, metallic, or ceramic) and, in some cases, a filler. One particular class of epoxy resins are the thermosetting plastics which enjoy prominence as potting compounds, adhesives, and surface coatings. For example, when binding graphite fibers in composite materials, epoxy resins yield light-weight structures with high tensile strength. In order to optimize the resulting material's strength properties, process control becomes a critical issue because the epoxy resin's final properties are strongly influenced by the type of resin and curing agent, the curing temperature, and the cure time.

In general, the cure of an epoxy involves a cross-linking reaction between an epoxy resin and a curing agent [2]. EPON 828, a bisphenol A-epichlorohydrin epoxy resin was cured in this investigation with the polyamide curing agent, Versamide 40.

The cross-linking reaction which occurs between a bisphenol Aepichlorohydrin resin and a polyamide is depicted in Figure 1. Initially, the epoxy resin and curing agent mixture is a liquid. As their chemical interaction proceeds, the epoxy resin is cross-linked into a network whose molecular weight is increasing. When a sufficient number of cross-links have been formed, the mixture forms a solid gel. This condition is referred to as the gelation point [3]. As the chemical reaction proceeds past the gelation point, the mixture forms a rigid, glassy solid, and the chemical reaction kinetics slow and eventually stop as the mobility of the polymerized reactants is decreased by the increasing viscosity of the glassy solid. The formation of the glassy solid is referred to as vitrification [3].

During the past decade, a number of diagnostic techniques have been developed to evaluate the degree of polymerization of epoxy resins. The more popular cure monitoring techniques include: infrared spectroscopy [4]; nuclear magnetic resonance spectroscopy [5]; torsional-braid analysis [6-8]; differential scanning calorimetry [5,7,9]; viscosity [10]; and hardness testing [11]. All of these techniques require the analysis to be accomplished in a well-equipped laboratory, destruction of the sample, and they are usually complex and costly to implement. However, the most serious limitation posed by these diagnostic techniques is that they cannot be readily be utilized to evaluate a product's in situ epoxy cure on a real-time basis.

Since epoxy resins and their curing agents are contaminated with ionic impurities, the electrical current flow through these materials consists of the conventional electrically polarized component, as well as an ionic component. It has been suggested that the ionic current conductivity will decrease as the curing process progresses because increasing polymerization will act to impede ion mobility [12]. As a result, a prominent solid-state sensor, referred to as the charge-flow transistor (CFT) or microdielectrometer, has been developed to monitor the cure of epoxy resins [13-17]. The monitoring of epoxy cure with the $\mathrm{CF}$ T is based on measuring the change in the material's complex dielectric function as it cures [14-17]. Usually, the epoxy cure data is reduced to a series of plots involving the dielectric loss factor (or alternating current conductivity) and the relative permittivity. An alternative perspective developed in this paper is to interpret the electrical conductivity associated with epoxy cure by utilizing the IGEFET and analyzing the sensor's temporally-dependent response in the frequency-domain.

\section{IGEFET SENSOR CONCEPT}

As illustrated in Figure 2, the IGEFET sensor consists of an interdigitated electrode structure which is coupled to the gate oxide of a conventional metal-oxide semiconductor field-effect 
transistor (MOSFET). The interdigitated gate electrode structure is composed of two interlaced electrodes: a driven- and a floatingelectrode. The driven-electrode envelopes the entire sensor and functions as the input to the IGEFET sensor and as a guard ring to minimize stray surface-leakage currents. The corresponding floatingelectrode component establishes electrical contact with the MOSFET's gate oxide. Electrical isolation between the driven- and floatingelectrodes (greater than $100 \mathrm{M} \Omega$ ) is accomplished by supporting them on a thick (at least 1 micron), high-quality (resistivity greater than $10^{14} \mathrm{ohm}-\mathrm{cm}$ ) silicon dioxide layer. The IGEFET's epoxy cure sensing capability is realized by depositing the epoxy mixture on the surface of, and between, the interdigitated electrode components. As the epoxy's molecular cross-linking reaction proceeds, its electrical impedance is perturbed. These impedance perturbations can be observed and quantified when the IGEFET's driven-electrode is excited with a voltage pulse. As a consequence of the floating-electrode being electrically connected to the MOSFET's gate oxide, charge is transferred through the curing epoxy and manifests itself as a temporally-dependent potential applied to the high-input impedance gate oxide contact of the MOSFET [13]. The judicious specification of the MOSFET's materials, geometry, and operating bias conditions establishes its amplification characteristics (principally, its gain, linearity, and cut-off frequency). As a result of the MOSFET's transfer function [18], the chemical reaction induced potential perturbation appearing at the MOSFET's gate terminal is manifested as a corresponding and amplified change in the device's drain-to-source current. By operating the MOSFET as a linear amplifier and connecting the drain-to-source terminals with an external series resistance, a potential corresponding to the temporally-dependent drain-to-source current can be directly measured across the resistor Thus, the electrical isolation afforded by the dielectric-supported interdigitated electrode and the gate oxide allows the MOSFET to manifest itself as an in situ "observation window" which is used to monitor the electrical behavior of the chemically-active epoxy while minimizing its influence on the dynamic molecular structure.

A significant limitation associated with current electronic thin film epoxy cure detectors is that they only examine the sensor's response as a single operating point; that is, a direct current resistance measurement or the impedance (complex dielectric function) at a specific frequency or limited set of frequencies. As a consequence, only a small portion of the material's impedance spectrum is utilized to monitor the progress of an epoxy's cure. Therefore, the IGEFET sensor is operated to minimize this limitation by observing the response in the frequency-domain.

The sensitivity of the IGEFET sensor to molecular structural changes is realized by computing the magnitude of a difference Fourier transform spectrum. That is, since the long-term excitation pulse characteristics were experimentally observed to be reproducible and time-invariant, the magnitude of the difference Fourier transform spectrum was computed by Fourier transforming the excitation pulse and the sensor's time-domain voltage response at specific time increments as the epoxy cures. The two spectra were then subtracted from each other on a point-by-point basis at common frequencies; the magnitude of the difference was used to generate the sensor's response envelope. In practice, the relative abundance of components which define the shape of the spectral response envelope yields valuable information for unambiguously specifying the state of the epoxy's cure. That is, as demonstrated experimentally, the magnitude of the difference Fourier spectrum clearly manifests the distinct dielectric relaxation behavior caused by the epoxy's cure and its resulting molecular structure.

\section{IGEFET SENSOR DESIGN AND FABRICATION}

The IGEFET was designed and fabricated using the Metal Oxide Semiconductor Implementation Service (MOSIS) $3-\mu \mathrm{m}$, p-well, doublemetal, complementary metal oxide semiconductor (CMOS) technology [19]. Except for a solid metal gate electrode, an otherwise identical reference transistor was located adjacent to each IGEFET sensor to facilitate post-fabrication MOSFET performance characterization and the subsequent monitoring of operational stability in the thermostated, epoxy-cure sample cell. A p-well was incorporated to enhance the electrical isolation between the interdigitated electrode structure and the MOSFET, and n-channel enhancement-mode devices were utilized. Aluminum was used to implement the second-level metal interdigitated electrode array and the bonding pads. Except for the interdigitated electrode structure and bonding pads, the surface of the IGEFET was passivated with a $3 \mu \mathrm{m}$ thick layer of silicon dioxide. The overall dimensions of the IGEFET integrated circuit was $4466 \mathrm{x}$ $6755 \mu \mathrm{m}$, and the critical dimensions are summarized in Table I.

\section{INSTRUMENTATION}

The electrical instrumentation arrangement for characterizing the cure of an epoxy-coated IGEFET is illustrated in Figure 3. A 4-volt peak amplitude, $50 \mu \mathrm{sec}$ duration, $256 \mathrm{~Hz}$ repetition frequency pulse excitation signal was applied to the IGEFET's driven-electrode using a function generator (Wave-Tek Corporation, model 148, San Diego, CA). The frequency spectra of the IGEFET's input excitation signal and output response were measured simultaneously with a lowfrequency ( $25 \mathrm{KHz}$ bandwidth), dual-channel Fourier transform analyzer (Bruel and Kjaer Instruments, Inc., model 2032, Marlborough, MA). To complement the frequency-domain measurements, the corresponding time-domain spectra were captured on a dual-trace oscilloscope (Tektronix Corporation, model 475 , Beaverton, OR). The impedance of the epoxy-coated interdigitated electrode structure was independently monitored with an impedance bridge (Hewlett-Packard Corporation, model HP4192A, Palo Alto, CA) whose frequency spanned $10 \mathrm{~Hz}$ to $10 \mathrm{MHz}$. A gain/phase analyzer (Hewlett-Packard Corporation, model HP4194A, Palo Alto, CA) was incorporated to measure the voltage gain $\left(20 \log _{10}\left[V_{\text {out }} / V_{\text {in }}\right]\right)$ and phase delay $\left(\phi_{\text {out }}-\phi_{\text {in }}\right.$ ) performance of the MOSFET portion of the IGEFET sensor and reference transistors using a sinusoidal test signal whose frequency spanned $10 \mathrm{~Hz}$ to $10 \mathrm{MHz}$. Data collection was automated using a microcomputer (Zenith Data Systems Corporation, model Z-248, St. Joseph, MI) equipped with an IEEE-488 interface plug-in card (Capital Equipment Corporation, model 01000-60300, Burlington, MA)

The temperature of the free-flow Pyrex sample cell was thermostated at $25 \pm 0.5{ }^{\circ} \mathrm{C}$ by submersing it in a water bath. Ultrahigh purity bottled nitrogen was used to establish a controlled ambient atmosphere throughout the epoxy cure cycle. A ground glass joint at the top of the sensor cell provided ports for the nitrogen gas delivery and exhaust, as well as the IGEFET's electrical wiring harness.

The procedure for monitoring the epoxy's cure was implemented as follows:

1. An isothermal baseline set of electrical measurements was accomplished by submersing the sample cell containing an uncoated IGEFET sensor into a constant temperature water bath thermostated at $25 \pm 0.5^{\circ} \mathrm{C}$. A dry nitrogen ambient whose flow was regulated at 1 liter/minute was allowed to freely pass through the sample cell.

2. An equal volume of EPON 828 and Versamide 40 were thoroughly mixed ( 2 minutes) and deposited on the interdigitated gate of the IGEFET sensor. A syringe with a 25-gauge hypodermic 
needle was used to apply the epoxy. The beveled edge of the needle was removed to improve control of the deposition rate. Small amounts of the epoxy were applied at several points along the length of the interdigitated array to reduce its lateral spread across the integrated circuit and prevent it from contacting adjoining interdigitated arrays.

3. The epoxy-coated IGEFET was then reinserted into the sample cell and purged with dry nitrogen whose flow was 1 liter/minute. The electrical performance measurements were initiated when the internal temperature of the sample cell equilibrated at $25 \pm 0.5^{\circ} \mathrm{C}$ and the relative humidity within the sample cell decreased to less than 10 percent (approximately 5 minutes).

4. Early in the epoxy cure cycle, the electrical performance measurements were repeated continuously. Two hours into the cure cycle, the measurements were repeated less often, approximately each 30 -minute increment.

\section{RESULTS AND DISCUSSION}

MOSFET Performance Characteristics. The electrical transfer function characteristics of the MOSFET portion of the IGEFET sensor and reference transistor were periodically monitored throughout the duration of the epoxy cure measurements. The average low-frequency gain for an ensemble of six uncoated MOSFET's measured with the gain/phase analyzer was $12 \pm 1 \mathrm{~dB}$. Independent confirmation of this value was derived from the direct current device characteristics measured with a semiconductor parameter analyzer (Hewlett-Packard Corporation, model HP4195, Palo Alto, CA). With a gate-to-source voltage $\left(\mathrm{V}_{\mathrm{GS}}\right)$ of 10 volts, the MOSFET transconductance $\left(\mathrm{g}_{\mathrm{m}}\right)$ was calculated to be $0.133 \mathrm{mS}$ from the slope of the drain current $\left(\mathrm{I}_{\mathrm{D}}\right)$ versus gate-to-source voltage $\left(\mathrm{V}_{\mathrm{GS}}\right)$ plot. Correspondingly, with a $\mathrm{V}_{\mathrm{GS}}$ of 2.5 volts, the drain-to-source on-resistance $\left(\mathrm{r}_{\mathrm{D}}\right)$ was calculated to be $130 \mathrm{~K} \Omega$ from the $I_{D}$ versus drain-to-source voltage $\left(V_{D S}\right)$ plot. Using these $g_{m}$ and $r_{D}$ values, along with the externally connected 100 $\mathrm{K} \Omega$ drain bias resistor (Figure 2), the direct current gain of the MOSFET was calculated to be approximately $15 \mathrm{~dB}$ [18]. By comparison, the gain of the reference MOSFET was consistently $1 \mathrm{~dB}$ greater than the gain of an adjacent IGEFET sensor, partially reflecting the differences between the two gate electrode geometries. Finally, the $3 \mathrm{~dB}$ cut-off frequency of the MOSFETs was on the order of $10 \mathrm{KHz}$, and the phase lag decreased from $180^{\circ}$ at low frequencies to nearly $90^{\circ}$ at $100 \mathrm{KHz}$.

Interdigitated Gate Electrode Impedance Measurements. The isothermal impedance measurements of the epoxy-coated interdigitated gate electrode structure revealed large perturbations in both the epoxy resistance and reactance during the polymerization process. The resistive and reactive components of the interdigitated gate electrode impedance during the epoxy's cure are illustrated in Figures 4 and 5 . The resistance and reactance information below $100 \mathrm{~Hz}$ are not shown because the magnitude of the impedance was not measurable (Hewlett-Packard Corporation, model HP4192A, Palo Alto, CA). Prior to epoxy deposition, the immeasurably low conductance of the ambient atmosphere above the electrodes and that of the silicon dioxide support below them produced a purely reactive interdigitated gate electrode impedance. When the epoxy was applied to the IGEFET, the resistive and reactive components, at any given frequency, decreased several orders of magnitude. The interdigitated gate electrode impedance was dominated by the reactive component over the entire frequency range. The largest relative contribution of the resistive component to the impedance occurs at approximately $40 \mathrm{KHz}$, and its contribution decreases uniformly at higher and lower frequencies. As time progressed and the epoxy cured, the magnitude of the interdigitated gate electrode resistive and reactive components increased, with the reactive component approaching the initial reactance of the uncoated interdigitated gate electrode structure. The relative contribution of the resistive component to the total impedance, particularly at higher frequencies, decreased as a result of the much larger increase in the reactive component.

IGEFET Time- and Frequency-Domain Measurements. The isothermal time-domain response of the IGEFET sensor to a voltage pulse excitation signal during the cure of the epoxy is depicted in Figure 6. The upper trace in each frame represents the 4 -volt peakamplitude, $50 \mu \mathrm{sec}$ duration, $256 \mathrm{~Hz}$ repetition frequency pulse excitation signal which was applied to the IGEFET's driven-electrode. The lower trace in each frame represents the IGEFET sensor's response prior to, during, and after the epoxy's cure $(0,106$, and 240 minutes). As shown in Figure 6a, the uncoated IGEFET sensor highly attenuates the excitation signal, especially at the higher frequencies, because of the $10 \mathrm{KHz}$ cut-off frequency of the MOSFET. The observed pulse distortion is characteristic of a low-pass filter response. In addition, when the epoxy was deposited, less of the excitation voltage signal was dropped across the interdigitated gate, and as a consequence, a larger signal reached the floating electrode. The larger signal on the floating-electrode was amplified by the MOSFET, and thus, the sensor's output voltage increased, as shown in Figure 6b. As noted earlier, as the epoxy cured, its impedance increased. This behavior results in a larger excitation voltage drop across the interdigitated gate electrode structure. Since the voltage drop on the floating-electrode has now correspondingly decreased, the IGEFET sensor's output voltage also decreases. The decrease of the IGEFET sensor's output voltage as the epoxy cured is shown in Figure 6c and $6 \mathrm{~d}$.

The corresponding Fourier transform difference spectra were computed for each of the associated time-domain measurements collected during the epoxy cure process. As shown in Figure 7, the uncoated IGEFET sensor's spectra reveals that the largest magnitude difference occurs at low frequencies. This characteristic implies that the IGEFET sensor is operating as a high-pass filter. When the epoxy is deposited on the interdigitated gate electrode structure, the IGEFET sensor's response increases, since less signal is dropped across the interdigitated gate. In turn this behavior had the effect of reducing the magnitude of the difference between the IGEFET sensor's output and the reference signal. The largest change in the difference spectra occurs at low frequencies, which corresponds to the higher conductivity manifested early in its cure cycle. As the epoxy continues to cure, less signal is dropped across the gate, and consequently, the IGEFET sensor's output is decreased, and the Fourier transform magnitude difference is correspondingly increased. The set of six curves in Figure 7 depict a smooth transition in the IGEFET sensor's response as the epoxy cures.

\section{CONCLUSION}

When utilized to monitor the cure of epoxy resins, the IGEFET sensor offers several advantages compared to the conventional parallel plate capacitance technique. First, its planar interdigitated electrode geometry is fixed and should be immune to those physical disturbances which may be encountered in the manufacturing environment. Second, because the IGEFET is an integrated circuit, its small size and mass afford the opportunity to unobtrusively implant it in a product being fabricated, or employed as a quality control device in samples weighing only a few milligrams. The inclusion of the MOSFET amplifier eliminates the need for electrical shielding and extends the utility of the IGEFET for detecting low-level signals extending over reasonably large bandwidths $(10 \mathrm{KHz})$.

The epoxy cure experiments motivated a novel signal processing technique which was employed to study the electrical conduction 
phenomena corresponding to the polymerization process. That is, the difference Fourier transform spectra yield valuable information regarding the behavior of the epoxy's low-frequency dielectric relaxation time, which has been previously correlated with the material's bulk viscosity properties. Consequently, it is postulated that the IGEFET sensor will increasingly serve a positive role as an in situ, real-time monitor of the viscosity of a curing epoxy system. Considering the importance of viscosity and its influence on the resulting physical properties of cured composite materials, the IGEFET is likely to assume a competitive position in the family of cure monitoring technologies.

\section{LITERATURE CITED}

1. Goldberg, B. and M. Semmel. Dielectric Cure Monitoring Preliminary Studies. National Aeronautics and Space Administration, George C. Marshall Space Flight Center, Huntsville, AL, NASA-TM86452, May 1984, available through the NTIS as document N64313787.

2. Miller-Stephenson Chemical Company, Incorporated. Epoxy Systems: Technical Information. Product Division, 1988, Danbury, CT.

3. Gillham, J. K. Polym. Engr. Sci., vol. 19, p. 676, 1979.

4. Acitelli, M.; R. B. Prime, and E. Sacher. Polymer, vol. 12, p. 335, 1971.

5. Larsen, D. W. and J. H. Strange. J. Polym. Sci., Polym. Phys. Ed., vol. 11, p. 1453, 1973.

6. Babayevsky, T. G. and J. K. Gillham. J. Appl. Poly. Sci., vol. 17, p. 2067, 1973.

7. Schneider, N. S. Polym. Engr. Sci., vol. 19, p. 304, 1979.

8. Gillham, J. K.; J. A. Benci, and A. Noshay. J. Polym. Sci. Symp., No. 46, p. 279, 1974.

9. Sourour, S. and M. R. Kamal. Thermochimica Acta, vol. 14 p. $41,1976$.

10. Tanaka, Y. and H. Kakiuchi. J. Appl. Polym. Sci., vol. 7, p. $1951,1963$.

11. Barton, J. M.; W. J. MacKnight and N. S. Schneider. Polymer Engr. Sci., vol. 19, p. 313, 1979.

12. Warfield, R. W. and M. C. Petree. J. Polym. Sci., vol. 37, p. 305, 1959.

13. Senturia, S. D.; C. M. Sechen, and J. A. Wishneusky. Appl. Phys. Lett., vol. 30, p. 106, 1977.

14. Sheppard, N. F.; S. L. Gaverick; D. R. Day; S. D. Senturia. Proc. 26th Nat. SAMPE Symp., pp. 65-76, April 1981.

15. Senturia, S. D. J. Adhesion, vol. 15, p. 69, 1982.

16. Senturia, S. D. SAMPE J., vol. 19, p. 22, 1983.
17. Senturia, S. D. and N. F. Sheppard. "Dielectric Analysis of Thermoset Cure", Epoxy Resins and Composites IV. K. Dusek (Ed.). Springer-Verlag: New York, 1986

18. Sze, S. M. Physics of Semiconductor Devices, 2nd ed. John Wiley and Sons: New York, 1981, Chapter 8, pp.431-455.

19. MOSIS - Metal Oxide Semiconductor Implementation Service User's Manual, release 3.0, 1988, Information Science Institute, Univeristy of Southern California (USC/ISI), Marina del Rey, CA.

\section{CREDIT}

The authors wish to acknowledge the financial and material support for this research provided by the United States Air Force, Air Force Systems Command, Wright Research and Development Center, Flight Dynamics Laboratory, Wright-Patterson Air Force Base, Ohio, under fund cite 616-88-114-364; and the Armstrong Aerospace Medical Research Laboratory, Wright-Patterson Air Force Base, Ohio, under fund cite HY-88-022
Table I

CRITICAL DIMENSIONS OF THE IGEFET SENSOR STRUCTURE
FEATURE

Interdigitated Gate Electrode

Number of fingers in the floating-electrode

Number of fingers in the driven-electrode 30

Electrode finger width $(\mu \mathrm{m})$

Electrode finger separation $(\mu \mathrm{m}) \quad 9$

Interdigitated electrode array length $(\mu \mathrm{m}) \quad 3792$

Interdigitated electrode array width $(\mu \mathrm{m}) \quad 921$ MOSFE'T

Active gate length $(\mu \mathrm{m})$

Active gate width $(\mu \mathrm{m})$ 


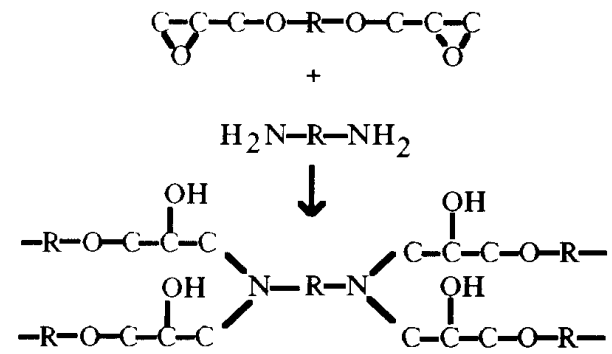

Figure 1. Cross-Linking Reaction Involving the EPON 828 Bisphenol A-Epichlorohydrin Epoxy Resin and the Versamide 40 Polyamide Curing Agent [2].

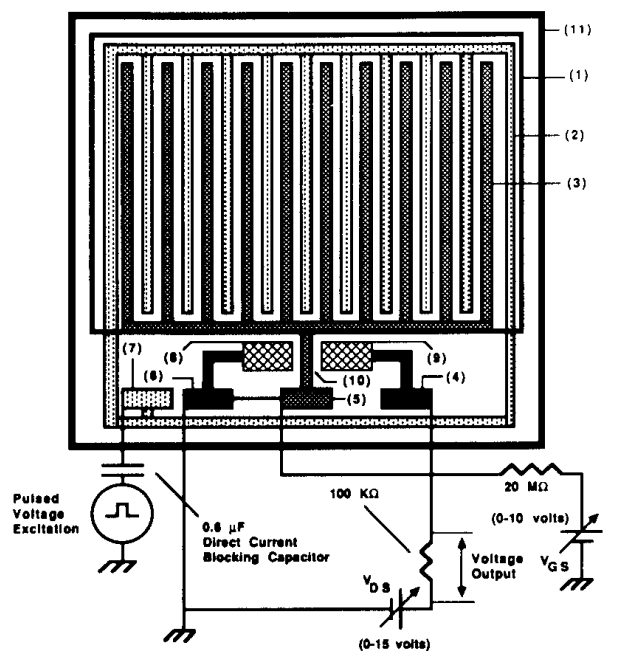

Figure 2. Interdigitated Gate Electrode Field Effect Transistor (IGEFET) Physical Structure and Electrical Connections. Legend: (1) Epoxy Resin, (2) Driven-Electrode Conductor, (3) Floating-Electrode Conductor, (5) MOSFET Gate and Floating-Electrode Contact, (6) MOSFET Source Contact, (7) Driven-Electrode Contact, (8) MOSFET Source Region, (9) MOSFET Drain Region, (10) Drainto-Source Channel, (11) Host Silicon Substrate.

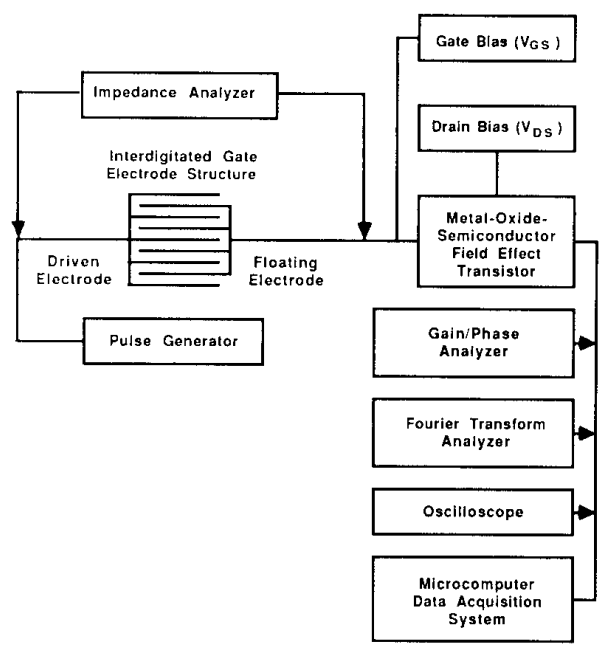

Figure 3. Instrumentation Arrangement for Measuring the Electrical Performance of the IGEFET Sensor.

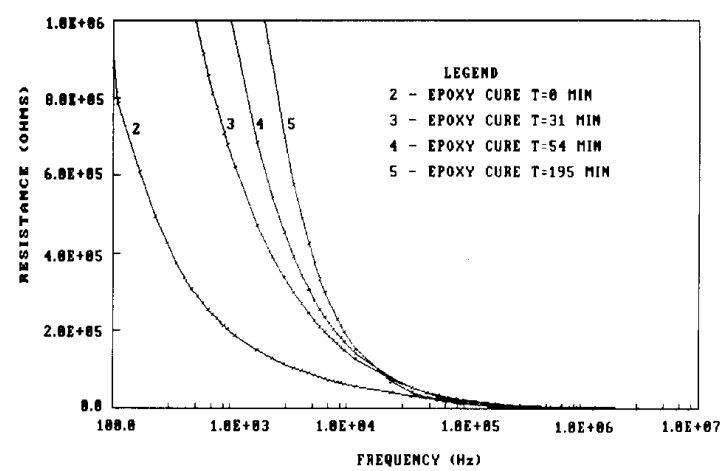

Figure 4. Resistive Component of the IGEFET Stizsor's Interdigitated Gate Electrode Impedance versus Frequency and as a Function of the Epoxy Cure Time [ultra-high purity nitrogen ambient $(1$ liter/minute flow) and $25 \pm 0.5{ }^{\circ} \mathrm{C}$ operating temperature]. 


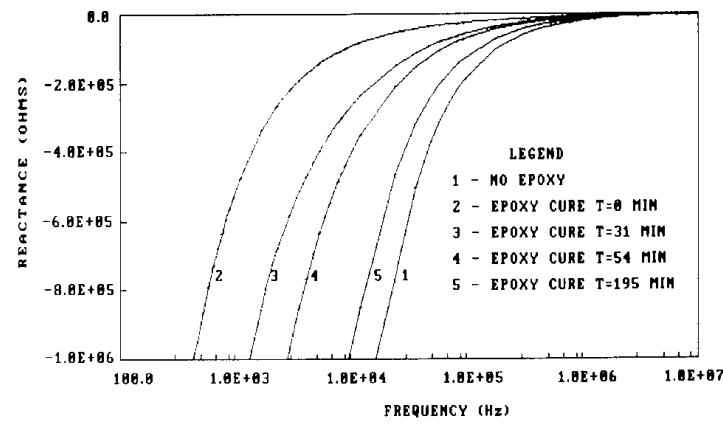

Figure 5. Reactive Component of the IGEFET Sensor's Interdigitated Gate Electrode Impedance versus Frequency and as a Function of the Epoxy Cure Time [ultra-high purity nitrogen ambient (1 liter/minute flow) and $25 \pm 0.5{ }^{\circ} \mathrm{C}$ operating temperature].

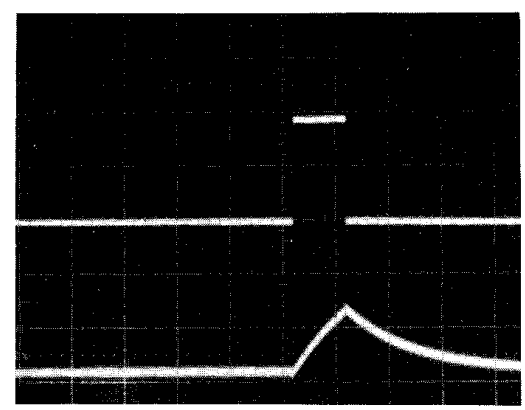

a)

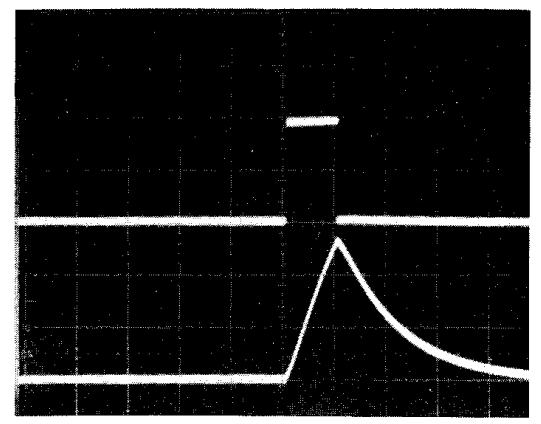

b)
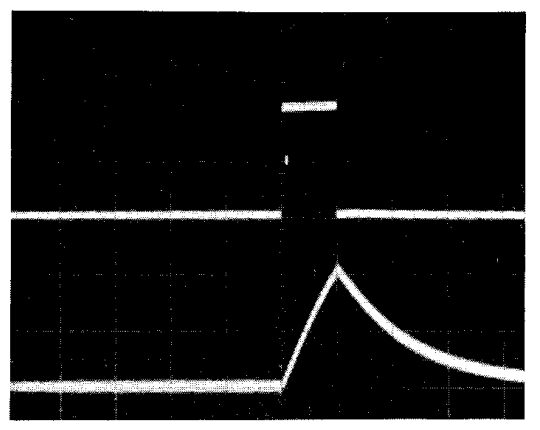

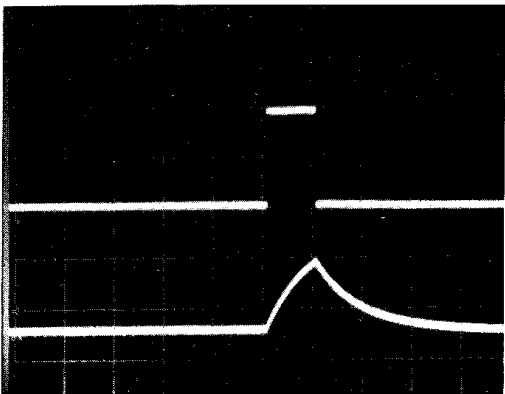

Figure 6. IGEFET Sensor Epoxy Cure Time-Domain Response when Excited with a Pulsed Voltage Waveform [ultra-high purity nitrogen ambient ( 1 liter $/$ minute flow) and $25 \pm 0.5{ }^{\circ} \mathrm{C}$ operating temperature]. Epoxy Cure Legend: a) Uncoated IGEFET Response

b) Epoxy Applied $(\mathrm{T}=0)$

c) Epoxy Cure ( $\mathrm{T}=106$ minutes)

d) Epoxy Cure ( $\mathrm{T}=240$ minutes)

The upper trace in each frame is the pulsed-voltage excitation signal (4-volt amplitude, $50-\mu$ sec pulse duration). The oscilloscope scale is 2 volts/major division (vertically), and $50-\mu \mathrm{sec} / \mathrm{major}$ division (horizontally). The lower trace in each frame is the sensor's response. The oscilloscope scale is: a) and b) 0.5 volts/major division (vertically, and $50-\mu \mathrm{sec} /$ major division (horizontally).

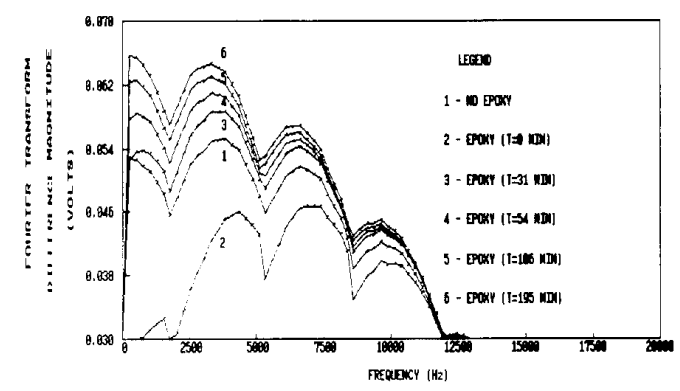

Figure 7. Fourier Transform Difference Spectra of the IGEFET Sensor's Response as a Function of Epoxy Cure Time [ultra-high purity nitrogen ambient ( 1 liter/minute flow) and $25 \pm 0.5^{\circ} \mathrm{C}$ operating temperature]. 